\title{
RESEARCH ON AUTOMATED DATA PROCESSING METHOD OF PCI GEOGRAPHIC IMAGING ACCELERATOR (GXL) SYSTEM FOR BIG DATA OF SUPERVIEW-1 REMOTE SENSING IMAGE
}

\author{
BAI Jin ${ }^{1}$, GAO Jingnan ${ }^{2} *$, DANG Yu ${ }^{1}$, LUO Fujun ${ }^{1}$ \\ ${ }^{1}$ National Quality Inspection and Testing Center for Surveying and Mapping Products, Beijing, China- 313219666@qq.com \\ ${ }^{2}$ SpaceWill Info. Co., Ltd, Beijing, China- jingnan Gao@spaceview.com
}

KEY WORDS: Remote Sensing, Big Data, Natural Resources, "Superview-1" Satellite Constellation, GXL System, Data Processing

\begin{abstract}
:
The Chinese government attaches great importance to the development of remote sensing satellites and has now formed satellite series such as meteorological satellites, ocean satellites, resource satellites and environmental disaster reduction satellites. At the same time, many commercial space programs have been put forward or implemented in China in recent years. As the first commercial-oriented multi-means and high-resolution optical remote sensing satellite constellation in China, "Superview-1" satellite constellation is undoubtedly one of the outstanding representatives. In the implementation of digital orthophoto map project, how to deal with large data of remote sensing image in large area quickly and accurately is a difficult problem we are facing to be solved urgently. PCI geographic imaging accelerator (GXL) system plays an important role in the production of digital orthophoto image by virtue of its advantages in mass image processing. This paper briefly introduces the structure and advantages of "Superview-1" satellite constellation, introduces in detail the production process and the key technologies of "Superview-1" satellite constellation image by GXL system, and carries out research on computational efficiency comparison and production test by GXL system. In this paper, the advantages of this software system in the production of tremendous amount of satellite remote sensing image data are analyzed. At the same time, in the existing engineering practice, due to the limitation of current data scale and production mode, the software system still has some obscure shortcomings. Under the background of natural resources, the amount of remote sensing data will continue to increase substantially in the future. By analyzing these shortcomings, this paper combines the traditional production mode with the background of remote sensing data in the new era, and puts forward some reasonable and useful suggestions.
\end{abstract}

2*Corresponding author: GAO Jingnan, E-mail: jingnan Gao@spaceview.com. 


\section{INSTRODUCTION}

In the 1960s, remote sensing technology rose in the world. Since the launch of China's first artificial earth satellite, China has entered the space age. China has made important achievements in the field of space flight, which attracts worldwide attention. In 2018, China's space launch activities reached a new record. A total of 39 launch missions were carried out in the year, accounting for more than one third of the global total; 37 of them were carried out by Long March series launch vehicles and 2 by other rockets; a total of 105 spacecrafts were launched, including 95 domestic and 10 foreign spacecrafts. Detailed information on launch of Chinese launch vehicles in 2018 can be found in Table 1. (China Aerospace Science and Technology Corporation, 2019) The Chinese government attaches great importance to the development of remote sensing satellites. It has formed a series of national satellites, such as meteorology, ocean, land resources, environmental disaster reduction, high scores and civil space infrastructure. At the same time, the privatization and commercialization of remote sensing have made considerable progress. Private satellite remote sensing has become an important supplement to the National Remote sensing system. (TONG Qingxi et.al, 2018) In 2018, China's commercial aerospace industry launched various commercial aerospace projects around satellite manufacturing, launch services, satellite operation, ground equipment and other industrial chains. The progress and breakthroughs achieved provide an important complement to China's aerospace system construction and capacity development, attract industry attention and market focus, and reflect Chinese tradition. The positive trend of continuous interaction between space flight and commercial space flight.

\begin{tabular}{|c|c|c|c|}
\hline $\begin{array}{c}\text { Development } \\
\text { departments }\end{array}$ & $\begin{array}{c}\text { Number } \\
\text { of } \\
\text { launches }\end{array}$ & $\begin{array}{c}\text { Number of } \\
\text { launching } \\
\text { spacecraft }\end{array}$ & $\begin{array}{c}\text { Launch } \\
\text { spacecraft } \\
\text { weight/kg }\end{array}$ \\
\hline $\begin{array}{c}\text { China } \\
\text { Aerospace } \\
\text { Science and } \\
\text { Technology } \\
\text { Corporation } \\
\text { China }\end{array}$ & 37 & 103 & 66136 \\
$\begin{array}{c}\text { Aerospace } \\
\text { Science and } \\
\text { Industry }\end{array}$ & 1 & 1 & 97 \\
Corporation & & 1 & 40 \\
Landspace & 1 & 105 & 66273 \\
Total & 39 & & \\
\hline
\end{tabular}

Table 1. Launch of Chinese Launch Vehicle in 2018

In the process of implementing digital orthophoto remote sensing image project, how to process massive images in large areas quickly and accurately is an important problem we are facing. The traditional method is to use Erdas, Envi, Pixel factory and other software to adjust, Ortho-rectifying and mosaic images. In the actual image production work, we can find that different software has different performance characteristics, but the same is that the number of images processed each time is very limited. And too much rely on manual intervention, automation is far from enough, which greatly affects the efficiency of our actual image production work. The PCI geographic imaging accelerator (GXL) system plays an important role in the production of Digital Orthophoto Image by virtue of its advantages in automatic processing of massive images. This paper briefly introduces the structure and advantages of "Superview-1" satellite constellation. According to the actual needs of the image data of "Superview-1" remote sensing satellite and the special features of GXL software system. At the same time, GXL software system is used to carry out the research on calculation efficiency comparison and production test, and the advantages and disadvantages of GXL software system in the production of Orthophoto images of massive remote sensing data are analyzed. In the context of natural 
resources, the amount of remote sensing data will continue to increase substantially in the future. Based on the analysis of these advantages and disadvantages, this paper combines the traditional production mode with the background of satellite remote sensing data of natural resources in the new period, and puts forward some reasonable and useful suggestions.

\section{INTRODUCTION OF "SUPERVIEW-1" COMMERCIAL REMOTE SENSING SATELLITE AND GXL SYSTEM}

\subsection{Introduction of "Superview-1" Commercial Remote Sensing Satellite}

In recent years, many commercial space programs have been put forward or implemented in China. As the first batch of commercial high resolution optical remote sensing satellite constellations, "Superview-1" satellite constellation is undoubtedly one of the outstanding representatives. "Superview-1" 01/02, on December 28, 2016, "Superview-1" 03/04, on January 9, 2018, two successful launches with one arrow and two satellites. The four satellites operate in the same orbit at an angle of 90 degrees, forming the SuperView-1 satellite constellation with a resolution of 0.5 meters, and the revisit period is shortened to 1 day."Superview-1" has panchromatic resolution of 0.5 $\mathrm{m}$, multispectral resolution of $2 \mathrm{~m}$, orbital altitude of $530 \mathrm{~km}$, width of $12 \mathrm{~km}$ and transit time of 10:30 a.m. Because "Superview-1" satellite has high agility, it can set multiple acquisition modes such as continuous strip, multi-strip splicing, shooting by target, and stereo acquisition. "Superview-1" can take $60 \mathrm{~km} \times 70 \mathrm{~km}$ images at a single shot. It has five core advantages: ultra-high resolution, optimized spectral band setting, wide shooting, outstanding agility and excellent acquisition ability.

\subsection{Introduction of GXL system}

PCI geographic imaging accelerator (GXL) system is the flagship product of Canada's PCI Geomatics Company. It integrates remote sensing image processing, GIS/spatial analysis, cartography and desktop digital photogrammetry system, and is an independent production platform. The system is mainly based on image data processing, especially remote sensing image processing. Its products involve remote sensing image processing (including hyperspectral and radar image processing), digital photogrammetry, spatial analysis, three-dimensional display, visual modeling and cartography, etc. With high industry influence, it is one of the advanced geospatial information processing software products in the world. With its abundant software modules, supporting all data formats, suitable for various hardware platforms, flexible programming ability and convenient data operability, it represents the development trend and technology pioneer of image processing system. It can be used not only in satellite and aerial remote sensing image processing, but also in geophysical data image, medical image, radar data image and optical image processing.

\section{THE PROCEDURE AND METHOD OF SATELLITE IMAGE MAKING}

The basic idea of using GXL system to process Superview-1 satellite image production experiment is: first, control point acquisition, regional network adjustment and ortho-correction of $0.5 \mathrm{~m}$ high-resolution image of experimental data source, then use the corrected $0.5 \mathrm{~m}$ high-resolution image to register $2 \mathrm{~m}$ multi-spectral image, after registration is completed. The purpose of this production experiment has been achieved by image fusion and mosaic of high-resolution and multi-spectral images. But in the routine production work, we still need to continue to color, cut and slice the mosaic image. Because the follow-up work is greatly affected by human intervention, so we will not express it in this paper. Figure 1 shows the experimental process of processing "Superview-1" satellite image using GXL system. 


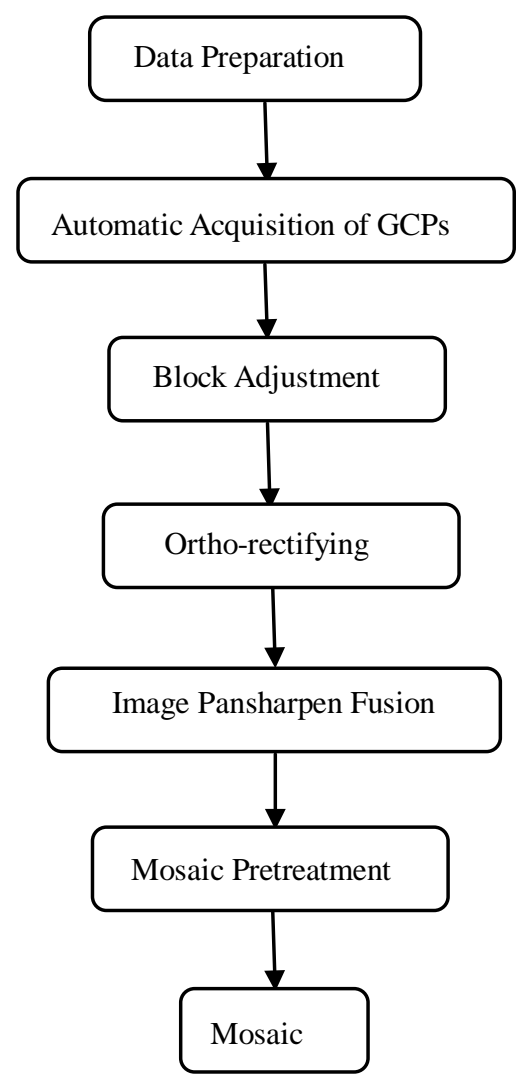

Figure 1. The Experimental Process of Processing

Satellite Image using GXL System.

\subsection{Data preparation}

First of all, the original data analysis is needed. Because the original "Superview-1" satellite image GXL cannot be recognized, it needs to be transformed into GXL recognizable format through image import. Using other programs, it is first converted into *. Pix format which can be recognized by GXL software, and then other operations are carried out.

\subsection{Automatic acquisition of GCPs}

Ground control point (GCP) acquisition is to collect the same name points of the input image by combining the reference image with DEM, so that the input image can be corrected to the reference image. At the same time, registration points are collected by registration algorithm to register the high-resolution image and multi-spectral image.

PCI supports automatic collection of GCPs from four kinds of reference data: ortho-correction image/geo-reference image, control point image database, line vector data (road network), polygon vector data (lake). The core technology of automatic GCP/TP acquisition is image correlation. It can judge the characteristics of reference image corresponding to the position of pixels and lines in the original image, or the reference position of image database and vector data. It should be noted that the search radius is set in pixels, and the conversion between pixels and meters in different resolution image sources should be noted.

\subsection{Block adjustment}

Block adjustment is to control the relative accuracy and absolute accuracy of images by collecting the connecting points between the overlapping areas of adjacent images and calculating the adjustment of the area with the control points of the previous acquisition step.

The control point mainly affects the absolute accuracy, while the connection point affects the relative accuracy. Because the absolute accuracy of "Superview-1" image itself is high, the matching between "Superview-1" image and reference image is generally good, so the relative accuracy of image is particularly important. Only when the relative accuracy is well controlled, can the whole large data area of remote sensing image reach a higher precision level. In the adjustment process of all adjacent images, special attention should be paid to the setting of adjustment parameters such as search radius, number of connection points (TP) and minimum score of TPs.

\subsection{Ortho-rectifying}

With the help of digital elevation model (DEM), the topographic deformation of each pixel in the image is corrected, so that the image meets the requirements of Orthophoto projection, which is the purpose of Orthophoto rectification. The process of GXL ortho-rectification module is that ortho-rectification of panchromatic image is performed first, then multi-spectral image is registered with panchromatic image, and ortho-rectification of multi-spectral image is carried out finally. The output result is panchromatic and multi-spectral ortho-image. The parameters of 
Orthophoto correction mainly focus on the following aspects: map projection, panchromatic pixel output size, multi-spectral pixel output size and pixel resampling type.

\subsection{Image Pansharpen fusion}

Due to the limitation of imaging principle and technical conditions, any single remote sensor cannot fully reflect all the characteristics of the target. That is to say, all kinds of remote sensors have their own limitations. If the data with two or more different characteristics are combined, the advantages of their respective images can be absorbed more comprehensively. Mapping objects on the ground can provide users with better information interpretation ability and greatly improve the value of image utilization. In this experiment, Superview-1 $0.5 \mathrm{~m}$ high resolution image and $2 \mathrm{~m}$ multi-spectral color image are fused by image fusion technology. The generated image has both high resolution and color information. The image fusion algorithm adopted by GXL is Pansharpen, which is an original and precise algorithm of GXL. Based on the mature least squares method, it seeks the best approximate gray value relationship among the original multispectral, panchromatic and fused images to achieve the best color performance. After integration, attention should be paid to whether the results spill over (ghosting).

\subsection{Mosaic pretreatment and Mosaic}

When the research area exceeds the coverage of a single remote sensing image, two or more images need to be stitched together to form one or a series. Columns cover larger images of the whole region, and this process is image mosaic. The GXL system performs all necessary pre-mosaic processing required to produce high-quality image mosaics, including color equalization, wiring generation, image standardization and image sequencing. Actually create the final output mosaic file.

\section{EXPERIMENTAL RESULTS AND PROBLEMS IN ACTUAL PRODUCTION}

\subsection{Experimental results}

In the experiment of processing large data of satellite remote sensing images with GXL system, the configuration parameters of the machine running GXL system are DELL Precision 5820T tower graphics workstation, CPU is Intel Xeon $\mathrm{W}-2145,3.70 \mathrm{GHz}$, memory is $32 \mathrm{G}$, and graphics card is NVIDIA Quadro P2000. In a recent experiment conducted from July 20 to 22, 2019, the processing time of GXL processing 350 images at a time is approximately 3.50 hours for image import, 9.00 hours for automatic acquisition of GCPs, 6.46 hours for block adjustment, 8.74 hours for ortho-rectification, 18.16 hours for mosaic pretreatment and mosaic. The total time was 45.86 hours. If we adopt the traditional PCI production method, the processing time of 35 images is 5 hours for automatic acquisition of GCPs, 2 hours for block adjustment and 2 hours for ortho-rectification, totally 8 hours. At the same time, due to the limitation of processing image scenes, we can only speculate that it takes about 80 hours to process 400 images.

Through the experimental results of GXL image processing, we can see that GXL system has obvious advantages in the process of processing large satellite image data, whether in terms of production data volume or production efficiency. There is no limitation on the number of images when GXL processes massive images. At present, the limitation is the configuration of machines and hard disk. Storage space. The greatest advantage of GXL is that it is automatic processing workflow from batch data import, automatic control point acquisition, automatic adjustment, automatic fusion, automatic orthographic, automatic mosaic, to automatic DEM extraction, and so on. It submits tasks by one key without manual operation.

\subsection{Problems in Practical Production}

In the actual project production application, the GXL system needs further improvement in some aspects: 
(1) The GXL system is not flexible and convenient enough to read data format. In the data reading link, the original file format of image data source needs to be converted to. pix file. In the Block adjustment link, the geodetic coordinates of control points need to be converted to longitude and latitude format.

(2) The non-uniformity of mining points may occur in GXL automatic acquisition control points, which is sometimes caused by control data. Sometimes, when facing individual topographic features, such as mountain areas, dams and other special topographic features, the accuracy will be poor due to fewer control points, and when large-scale changes occur, the accuracy will also be poor. Be careful, Processing method can use Erdas and other software to do manual dotting, but also pay attention to controlling the timeliness of data and so on.

(3) When GXL ortho-rectification is carried out, it is impossible to expand the image according to the user's needs. Occasionally, there will be dissatisfaction with the whole scene rectification in the operation.

\section{CONCLUSIONS}

(1) Based on the existing control data, it is reliable and efficient to use GXL system distributed processing technology and automatic processing to produce Orthophoto Image Based on "Superview-1" remote sensing image, which provides a good technical support for the rapid processing of large area remote sensing big data.

(2) GXL system is a powerful image processing system. It can satisfy the automatic production requirement of big data of mass remote sensing image for users.. The WEB user interface is simple and easy to operate. It supports multi-CPU, GPU acceleration and distributed processing. For the automatic processing system of massive satellites, it is not limited by the size of satellite data, and supports massive data. According to the automatic batch generation, the automation degree is higher, and user-defined workflow can be realized. This method breaks the traditional mode of operation, greatly reduces the time of manual intervention, and makes it possible to process large remote sensing data quickly. These advantages are incomparable to most remote sensing software at present.

(3) Prospects in the context of natural resources. With the establishment of the Ministry of Natural Resources, it is faced with natural resources such as land, minerals, forests, grasslands, wetlands, water and oceans. Whether the production of basic data or the investigation, analysis and evaluation work, the demand for remote sensing image will increase day by day. The amount of satellite remote sensing image data that surveying and mapping engineers will face in the future will also increase geometrically. In the era of big data, there is still room for improvement in the production mode of remote sensing image. It can better serve the work of natural resources system.

\section{ACKNOWLEDGEMENTS}

This study is sponsored by National Natural Science Foundation of China (41671440).

\section{REFERENCES}

China Aerospace Science and Technology Corporation, 2019. Blue Paper on China's Space Science and Technology Activities. Space International, (02),5-11.

Tong Qingxi, Meng Qingyan, Yang Hang, 2018. Development and Future Prospect of Remote Sensing Technology. Remote Sensing and Disaster Reduction, (06),2-11.

Wu Xiaotian, Xu Kang, Wang Yanmin, 2015. Research on Orthophoto Production of Massive Remote Sensing Data Based on GXL. Modern Surveying and Mapping, 38(04),23-25

Wang Jiongjie, 2018. Application of PCI GXL System in the Third National Land Survey on the Production of Positive Projective Images. Mine Surveying, 46(04),85-88. 
Xu Zhenbei, Li Aiyong, Yan Yong, 2017. Data Processing of Tiantu-1 Satellite Image Based on GXL. Geomatics \& Spatial Information, 40(10),86-88.

Jia Jie, Huang Mingying, Wan Junxin, 2018. Research on Geographic Situation Monitoring Panoramic Image Correction Technology Based on PCI GXL. Geomatics \& Spatial Information, 41(12),129-132.
Su Kai, Liu Meilin, 2017. Research on Fast Orthophoto Correction of Domestic Satellite Images Based on PCI GXL. Mine Surveying, 45(03),38-41.

Dou Xiaonan, Zhu Lin, Xie Mengli, 2016. Standard Time Point Approval for Geographic and National Conditions Census of Zhumadian City Based on PCI GXL. Beijing Surveying and Mapping, (02),1-4. 